\title{
Flight Test of a Head-Worn Display as an Equivalent-HUD for Terminal Operations
}

\author{
Shelton, K.J., Arthur III, J.J., Prinzel III, L.J., Nicholas, S.N., Williams S.P., and Bailey, R.E. \\ NASA Langley Research Center, Hampton, VA 23681
}

\begin{abstract}
Research, development, test, and evaluation of flight deck interface technologies is being conducted by NASA to proactively identify, develop, and mature tools, methods, and technologies for improving overall aircraft safety of new and legacy vehicles operating in the Next Generation Air Transportation System (NextGen). Under NASA's Aviation Safety Program, one specific area of research is the use of small Head-Worn Displays (HWDs) as a potential equivalent display to a Head-up Display (HUD). Title 14 of the US CFR 91.175 describes a possible operational credit which can be obtained with airplane equipage of a HUD or an "equivalent" display combined with Enhanced Vision (EV). A successful HWD implementation may provide the same safety and operational benefits as current HUDequipped aircraft but for significantly more aircraft in which HUD installation is neither practical nor possible. A flight test was conducted to evaluate if the HWD, coupled with a head-tracker, can provide an equivalent display to a HUD. Approach and taxi testing was performed on-board NASA's experimental King Air aircraft in various visual conditions. Preliminary quantitative results indicate the HWD tested provided equivalent HUD performance, however operational issues were uncovered. The HWD showed significant potential as all of the pilots liked the increased situation awareness attributable to the HWD's unique capability of unlimited field-of-regard.
\end{abstract}

Keywords: Head-Worn Display, HWD, Head-Up Display, HUD, Enhanced Vision, EV, Equivalent HUD, NextGen

\section{INTRODUCTION}

\subsection{Overview}

A HUD is a display system which projects information onto an optical-quality piece of semi-transparent glass (i.e., the combiner) fixed to the vehicle directly between the operator and the windscreen in front of the operator. The information is projected in a fixed field-of-view from an image generation source and collimating optics. HUDs have many benefits in commercial and business aircraft operations, providing an eyes-out, conformal display of critical flight parameters/symbology. As such, HUD operations improve safety by reducing the visual accommodation and re-accommodation that the pilot would otherwise have between reading head-down instrumentation and transitioning to out-the-window visual references. The safety benefits of the HUD were quantified in a 2009 study [1] which found that HUDs could have prevented or positively influenced $38 \%$ of accidents overall in aircraft equipped with modern glass cockpits.

The explicit display of flight path and energy information on the HUD promotes precision touchdown capability and energy management - i.e., maximizing landing performance (minimizing aircraft wear-and-tear). Studies show that significantly lower touchdown sink rates are flown under manual than automatic landings [2], and experimental evidence show that the HUD promotes better sink rate control and touchdown performance [3] [4]. Despite these advantages, HUDs are not standard equipage, although their prevalence is increasing. More than 3,300 HUDs are in service worldwide from one manufacturer alone, and are certified on 40 air transport category aircraft [5], including the Boeing 787, equipped with dual HUDs as standard equipment.

Thanks in part to the characteristics of the HUD, and in addition to safety benefits, "operational credits" are now being derived from HUD equipage, including:

- Fail-passive landing capability to 50-foot DH and RVR of 600-foot using HUD-driven guidance through approach, flare, landing, and roll-out

- Low visibility takeoff minima of 300-foot RVR

- Special Category II minima on Type I ILS of 100-foot DH, 1,200-foot RVR

- Reduction in Category II minima to 1,000-foot RVR; and

- Special Category I minima of 150-foot DH, 1400-foot RVR in lieu of centerline and touchdown zone lighting. 
Currently, the HUD is the only display certified and approved for use as part of an Enhanced Flight Vision System (EFVS). With EFVS - the combination of an imaging system and HUD - a pilot may descend below the published decision altitude (DA) from a straight-in instrument approach using an EFVS in lieu of natural vision (as per Federal Aviation Regulation $\$ 91.175$ (l) and (m)).

\subsection{HWD as HUD-Equivalent Display}

Within the "EFVS rule", regulations make provision for an equivalent display. Specifically, $§ 91.175$ (m) states that the Enhanced Flight Vision System (EFVS) sensor imagery and aircraft flight symbology must be presented “... on a head-up display, or an equivalent display, so that they are clearly visible to the pilot flying in his or her normal position and line of vision and looking forward along the flight path ..." In other words, an equivalent display must be some type of head-up presentation of the required information. A Head-Down Display (HDD) does not meet the regulatory requirement.

A critical component of EFVS performance is the integration of the "visual-like" imagery with symbology where the imagery is a display of the external scene from an imaging sensor, such as a Forward-Looking Infra-Red (FLIR) or millimeter wave radar. The primary reference for maneuvering the airplane is based on what the pilot sees through the EFVS and the conformal HUD symbology. As such, the required external visual references must be continuously and distinctly visible and identifiable by the pilot.

\subsection{HWD Requirements}

NASA has conducted research identifying requirements for a HWD to be an equivalent display to a HUD [6] as simply: Minimal user encumbrance - no more weight or encumbrance than current aviator's head-worn equipment (e.g., sunglasses and headset), and equivalent optical performance to existing HUDs. Research and development is on-going with respect to quantifying requirements for "minimal user encumbrance" although at this time, none are available. Optical performance requirements for a HWD to meet a "HUD-equivalent" capability are derived from FAA guidance [7] material specific to EFVS operations and minimum performance standards published by SAE and other organizations [8] [9].

The tracker-coupled HWD provides conformal symbology and imagery on a see-through, Near-To-Eye (NTE) display. The pilot freely moves his/her head, providing unlimited field-of-regard viewing of real-time symbology and imagery conformal to the outside environment.

\subsection{HWD Flight Test}

A proof-of-concept flight test was conducted to evaluate the maturity of technology undergoing development and evaluation at the NASA Langley Research Center (LaRC) and generate data to support industry and government guidance for HWD development for commercial and business aircraft applications. The HWD used monochrome HUD-type symbology and imagery in the forward looking, bore-sight direction to be analogous with a certified HUD with EFVS capability (Figure 1, right); that is, a virtual HUD or HUD-equivalent concept. We also provided off boresight conformal symbology in any direction the pilot moved his head. A FLIR camera was not available for the flight test; thus, a monochrome visible light camera was used to simulate enhanced vision imagery.
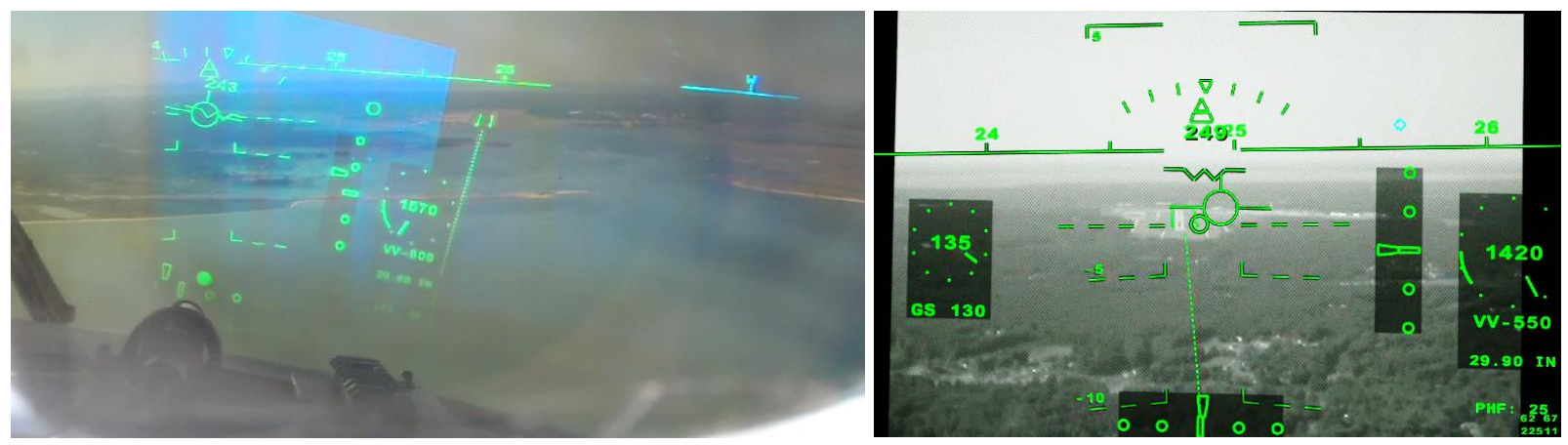

Figure 1: On the left, pilot's view through the HWD as the pilot is looking $~ 15$ degrees right of bore-sight direction while turning on final approach. On the right, a HWD snapshot showing HUD type symbology and enhanced vision imagery when pilot is looking directly forward along the bore-sight direction on the ILS approach. 
The pilot in Figure 1 (left snapshot) was looking approximately 15 degrees right of bore-sight while turning on final approach. Notice the runway depiction with extended centerline outside of the virtual HUD area. The off bore-sight symbology included the conformal representation of: the destination airport runway outline with a 10 mile extended centerline, a 360 degree horizon line with heading ticks, and air traffic indications. Non-standard HUD symbology were displayed in color: traffic was cyan diamonds and the off bore-sight horizon line was rendered in white. In Figure 1Error! Reference source not found., the cyan diamonds indicates traffic aircraft. The symbol size was proportional to proximity. The dark areas around the FLIR imagery were clear on the HWD allowing unobstructed vision of the outside world.

\subsection{Significance}

Extending the significant safety advantages of HUDs [1] to a much larger set of users is the primary operational significance that results from the addition of HWDs. HWDs also have several implementation advantages as compared with HUDs: HUD installation is not possible or practical by volume or weight in many new aircraft; HUD retro-fit is not cost-effective; and HWD installation has a return-on-investment advantage simply due to the substantially greater weight of current HUD systems. If HWD equivalence is successfully demonstrated and implemented, not only will HUD equivalence capabilities and credit be provided, but the significant off-bore-sight capabilities could be exploited with and for new applications. HWDs have potential for much lower implementation costs, and could be applied to more aircraft due to its smaller size and weight which makes retro-fitting to the existing fleet of aircraft feasible.

\section{METHODOLOGY}

A flight test was conducted to assess the HWD state of technology for "HUD equivalence" in the flight environment.

\subsection{Head Worn Display System}

The HWD was a custom-made and ruggedized combination of off-the-shelf Lumus DK-32 display glasses, as shown in Figure 2, and a prototype head tracker. The Lumus eye-wear is a see-through, full color display which utilizes patented Light-guide Optical Element (LOE) technology to generate an image that appears at "practical" infinity. The total head-born weight of the display, tracker, and a portion of the cabling is 7 oz. (202 grams).
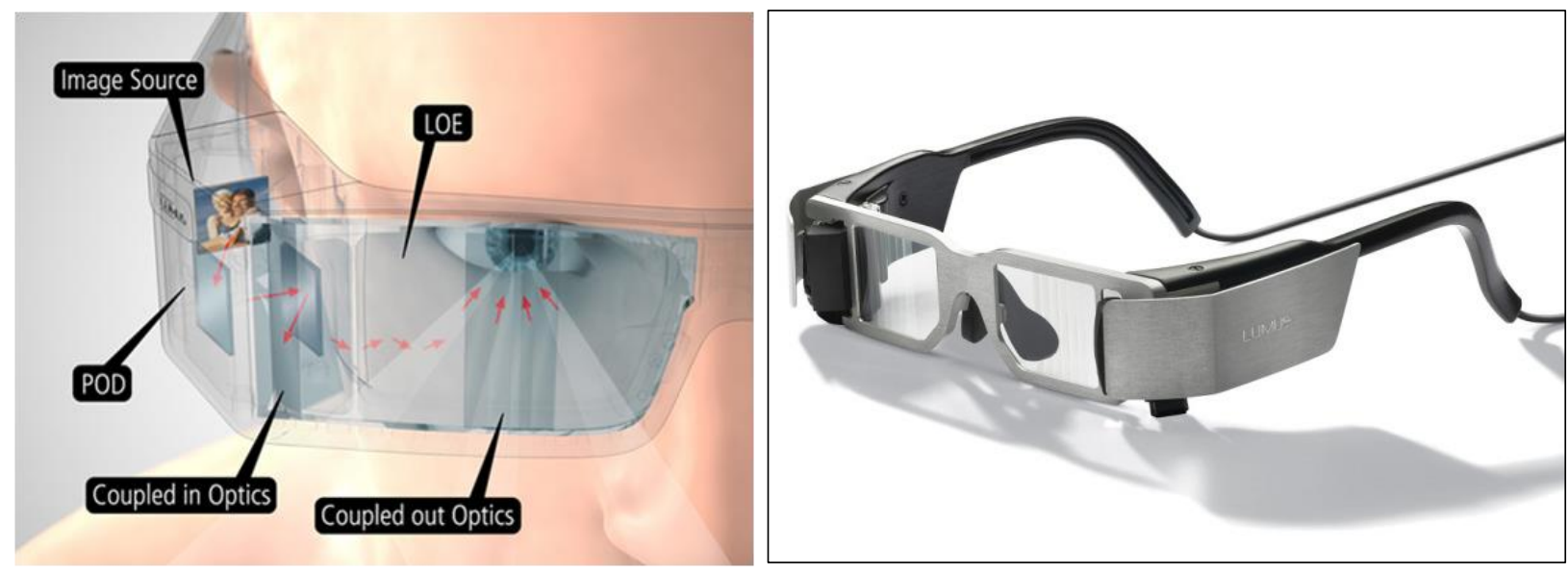

Figure 2: Lumus DK32 HWD with Light-guide Optical Element technology

The DK-32 has a resolution of $1280 \times 720$ pixels, 35 degree horizontal field-of-view, 20 degree vertical field-of-view, a maximum brightness of $1000 \mathrm{fL}$., and the image focal plane is at infinity. As the maximum brightness of the HWD was not bright enough for readability in daytime conditions, dark filters (essentially clip-on sunglasses) were used to increase the contrast of the HWD. This was sufficient for flight testing but a certified system would require a much brighter HWD.

\subsection{Head Tracker}

The advanced, prototype head tracker developed by Intersense (now Thales Visionix, Inc), shown in Figure 3, was mounted on the right side of the HWD. The head tracker is a hybrid (inertial, optical) system which uses inertial 
sensors for position and attitude tracking, and an optical system that corrects for the inertial drift by use of an infrared camera and illumination light emitting diodes (LEDs). The camera tracks small targets, called fiducials that are placed in the aircraft flight deck. These targets are retro-reflective stickers with unique identification. They are placed in the field-of-view of the camera, which was over the windscreen, center and side post and side windows. The fiducials are mapped in 3-dimensional space for the image processing to correct inertial drift.
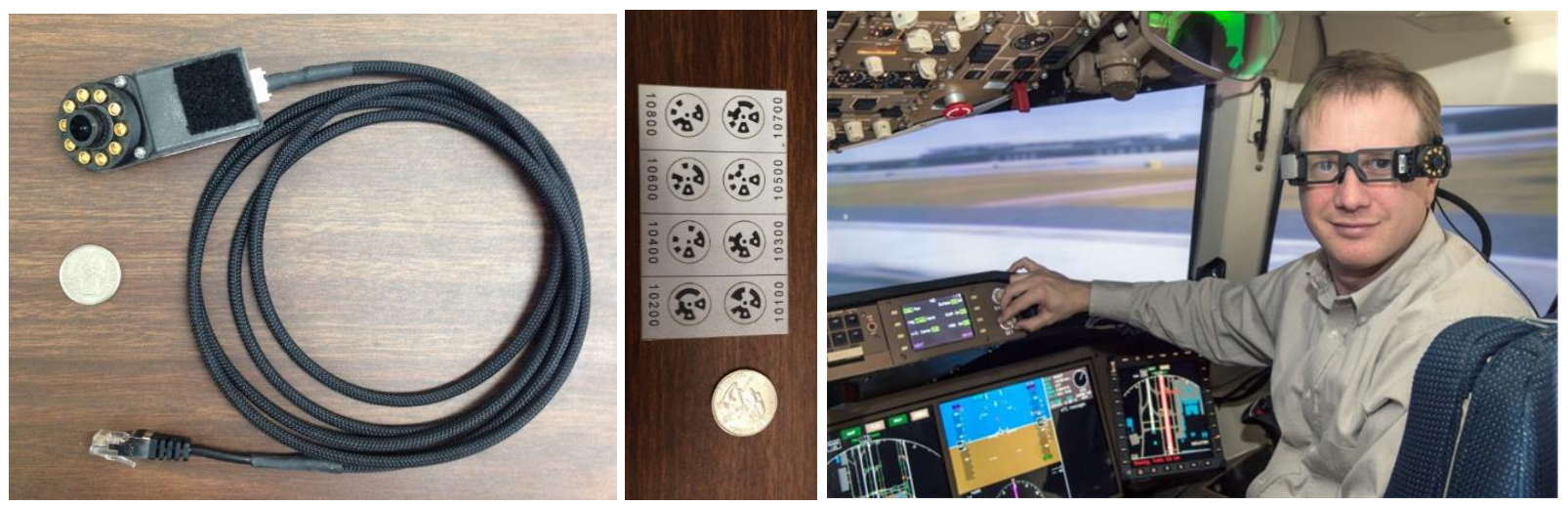

Figure 3: Head Tracker, fiducial targets, and Lumus DK-32 HWD with Intersense Head Tracker mounted on left side

The complete HWD system as tested included: the NTE display glasses, a head tracking system, a pseudo-EV image source (analog RS-170 camera), and an image generator (display computer) which interfaces to aircraft systems. An operator console was used by the researchers to operate the HWD system. A display computer interfaced to several digital signal sources including: the Air Data Unit providing altitude and airspeed data, the Inertial Measurement Unit (IMU) providing aircraft state data, the Intersense Head Tracker providing real-time pilot head position, an Automatic Dependent Surveillance-Broadcast (ADS-B) radio providing traffic position, and a visual camera mounted on the glare shield providing a real-time but simulated FLIR image.

\subsection{Test Method}

The HWD evaluations were flown in the NASA Langley Beechcraft King Air (BE-200) aircraft. The BE-200 is a corporate sized twin turbine aircraft that can be flown single pilot. Approach speed and procedures were similar to commercial operations. The flight test crew included: NASA safety pilot in the left seat, evaluation pilot (EP) in the right seat, a system operator, test director / researcher, and up to two (2) observers in the cabin.

Shown in Figure 4, a bore-sight reticle unit (BRU) was mounted on the aircraft glare shield to align the HWD display to the aircraft. The procedure for bore-sighting was part of the normal HWD operations for the EPs and was simple to perform at any time during the flight. The EV component of the HWD was simulated by use of a "lipstick" size visual camera mounted on the glare shield next to the BRU, and represented a real-time FLIR raster source that was integrated with aircraft state symbology. Brightness controls were mounted in the overhead to allow the EPs to control the overall HWD brightness, the EV imagery brightness, and a pushbutton to bore-sight the HWD. 


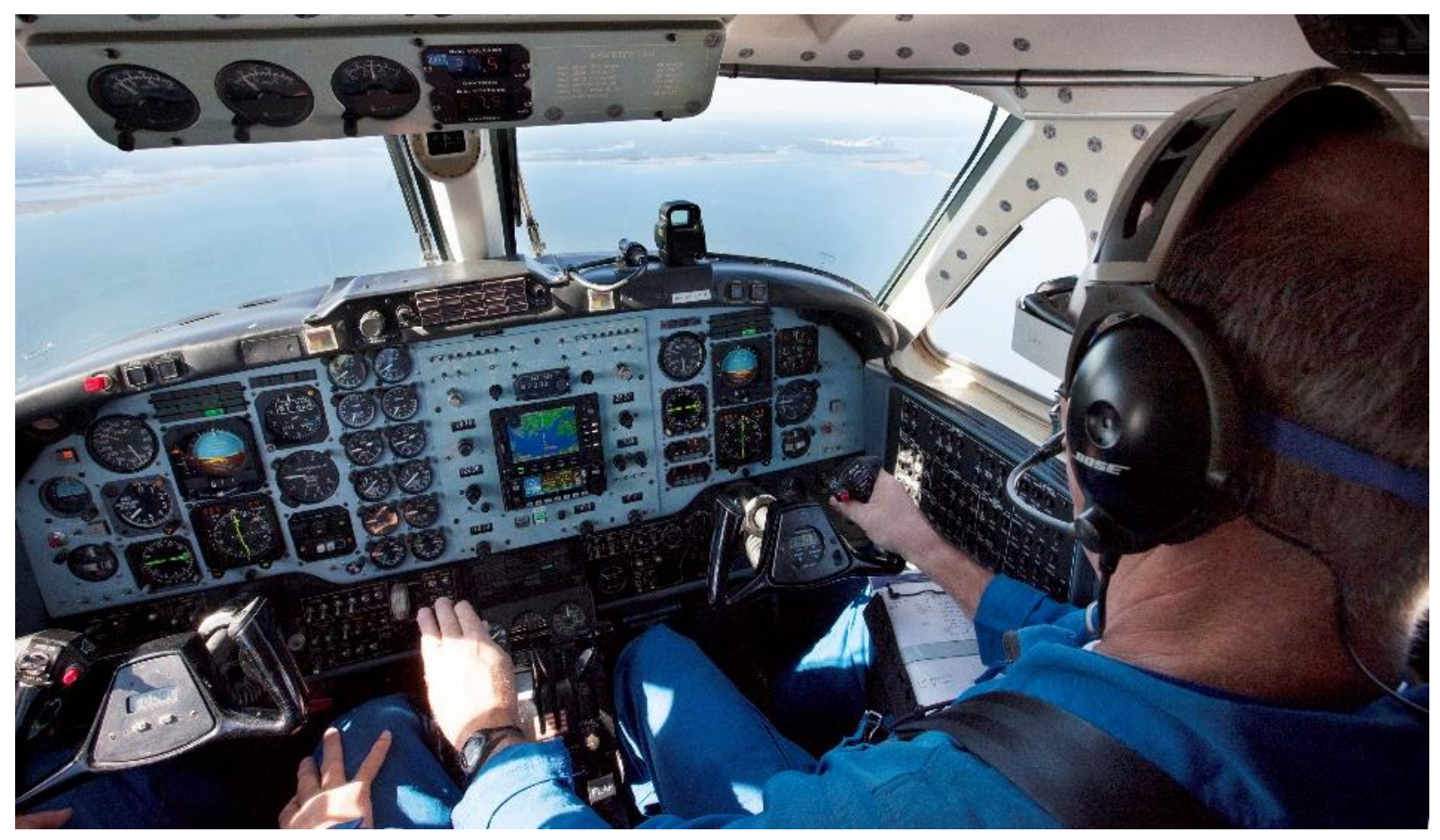

Figure 4: BE-200 Cockpit with Evaluation Pilot in Right Seat wearing the HWD

\subsection{Evaluation Subjects}

Seven flight test pilots served as EPs and were recruited based on the following selection criteria:

- Each EP held an Airline Transport Pilot rating or equivalent and trained or holds equivalent experience and served as a test pilot;

- Each EP had HUD and/or Helmet Mounted Display (HMD) experience, having flown at least 100 hours of HUD or HMD, pilot-in-command operations;

- Each EP had EV/EFVS experience, either military, general aviation, or commercial;

- Each EP had 20/20 visual acuity; if correction required, only correctable by use of contacts (no glasses as they were not compatible with the HWD tested);

The test pilots were from industry (corporate and commercial), military, and NASA civil service background. Average flying experience was more than 20 years, greater than 9000 flight total hours, and greater than 1000 hours HUD experience.

\subsection{Flight Test Operations}

NASA safety protocols prohibited the evaluation pilot from operating aircraft controls during take-off and landing. Therefore, the safety pilot conducted all take-off and landing maneuvers while the evaluation pilot observed the operations with the HWD. The evaluation pilot operated the flight controls in all other phases of testing. All test operations were conducted in Visual Meteorological Conditions (VMC) from the NASA Langley Research Center. Instrument Meteorological Conditions (IMC) were simulated via commercial off the shelf IMC training clip-on vision blockers. The ILS approaches were flown by the evaluation pilot down to $200 \mathrm{ft}$. above touchdown height. Approach guidance was provided as single cue pursuit guidance on the HWD. All the approach operations were conducted at Newport News International Airport (KPHF) and Langley Air Force Base (KLFI).

\subsection{Test Matrix}

Test operations included taxi and ILS approach scenarios designed to evaluate HWD performance and structured following existing SAE HUD performance requirements and Minimum Aviation System Performance Standards (MASPS) for EFVS (DO-315). The test controlled two independent variables; the evaluation pilot's presence/absence of natural vision (IMC/VMC) and the display of enhanced vision imagery (FLIR ON/OFF). The visibility was set to 
either VMC or simulated IMC, by use of a flip-down view-limiting device identical to the traditional clip-on instrument training aid. This device blocked the evaluation pilots view out the window but still allowed full use of the HWD and head down instruments. The enhanced vision imagery (simulated FLIR) was electronically enabled as necessary per the run cards for the evaluation subject.

Table 1: Test Matrix

\begin{tabular}{|c|c|c|c|c|}
\hline \multirow{2}{*}{ Test Run } & \multicolumn{2}{|c|}{ VMC } & \multicolumn{2}{c|}{ IMC } \\
\cline { 2 - 5 } & $\begin{array}{c}\text { FLIR } \\
\text { On }\end{array}$ & $\begin{array}{c}\text { FLIR } \\
\text { Off }\end{array}$ & $\begin{array}{c}\text { FLIR } \\
\text { On }\end{array}$ & $\begin{array}{c}\text { FLIR } \\
\text { Off }\end{array}$ \\
\hline \hline Taxi & $\checkmark$ & $\checkmark$ & & \\
\hline Approach & $\checkmark$ & & $\checkmark$ & $\checkmark$ \\
\hline
\end{tabular}

\subsection{Evaluation Tasks/Actions}

The evaluation pilot's task was to operate the aircraft as the "pilot flying" in a two person crew. The safety pilot acted as the "pilot not flying" and handled the radio and ancillary tasks as well as executed tasks at the request of the evaluation pilot. The evaluation of several aspects of the HWD were requested including: general head tracker operation during maneuvers, system latency, symbology and imagery conformance, and display optical performance in variable lighting.

The testing was conducted in four phases on each sortie:

1) Ground Test: On-Ramp, after engine-start, donning and bore-sighting, the EP evaluated the system performance of the HWD and gave an initial impression of the device while the aircraft was still in the chocks.

2) Taxi Trials: Two taxi trials were conducted - the initial taxi-out, and the return taxi-in after the flight. The FLIR runs were enabled randomly across subjects. Run questionnaires were issued after each trial.

3) Flight Trials: Three approaches were conducted by the evaluation pilots in VMC and simulated IMC conditions, with and without FLIR. Run conditions were randomized across EPs. Run questionnaires were issued after each trial.

4) Post-Test: Following completion of the in-aircraft testing, post-test surveys and interviews were conducted to capture summary pilot comments, ratings, and assessment.

\subsection{Questionnaires}

After each trial run, EPs rated their situational awareness via 3-point Situation Awareness Rating Technique (SART) [10], workload via the Air Force Flight Test Center (AFFTC) [11] 7-point workload scale and to rate their agreement (1-Strongly Disagree to 7-Strongly Agree) with statements on readability, comfort, usability, imagery, symbology, field-of-view, obscuration, impairment, and conformality. After the flight, the evaluation pilots were given a questionnaire asking their ratings on comfort, optical performance, bore-sighting procedures, brightness, color shifts, distortions, conformality, FLIR usage, eye strain, headaches, perceived safety, safety compared to HUDs, and equivalency to HUDs.

\section{RESULTS}

The purpose of the flight test was primarily to evaluate the use of HWDs during actual aircraft taxi and approach operations but also yielded dependent measures that provided descriptive statistics and quantitative and qualitative statistical results. A typical data flight lasted approximately 1.5 hours of flight time.

\subsection{Quantitative Results}

Flight technical error (FTE) data was collected on the approach from $1000 \mathrm{ft}$. to the controls handoff at approximately $200 \mathrm{ft}$. The box plot in Figure 5 illustrates the maximum localizer deviation across all runs in both the visual conditions (IMC/VMC) and with both FLIR conditions (on/off). The box plot in Figure 6 illustrates the maximum glideslope deviation across all runs in both the visual conditions (IMC/VMC) and with both FLIR conditions (on/off). The box plots show the median (solid line), $25^{\text {th }}$ and $75^{\text {th }}$ percentiles by the extent of the box, and maximum and minimum data 
extent by the whiskers. Statistical outliers are indicated by the star points beyond the extent of the whiskers as they have exceeded 1.5 times the inner-quartile range (i.e., either the 25th or 75th for low and high whisker).

The data in Figure 5 shows that the evaluation pilots were able to track the localizer well within $+/-0.1$ dots since the median and $75^{\text {th }}$ percentile maximum excursion was less than $+/-0.1$ dots and the maximum recorded excursion was just barely outside that value for all conditions. One outlier occurred in the FLIR On, IMC condition.

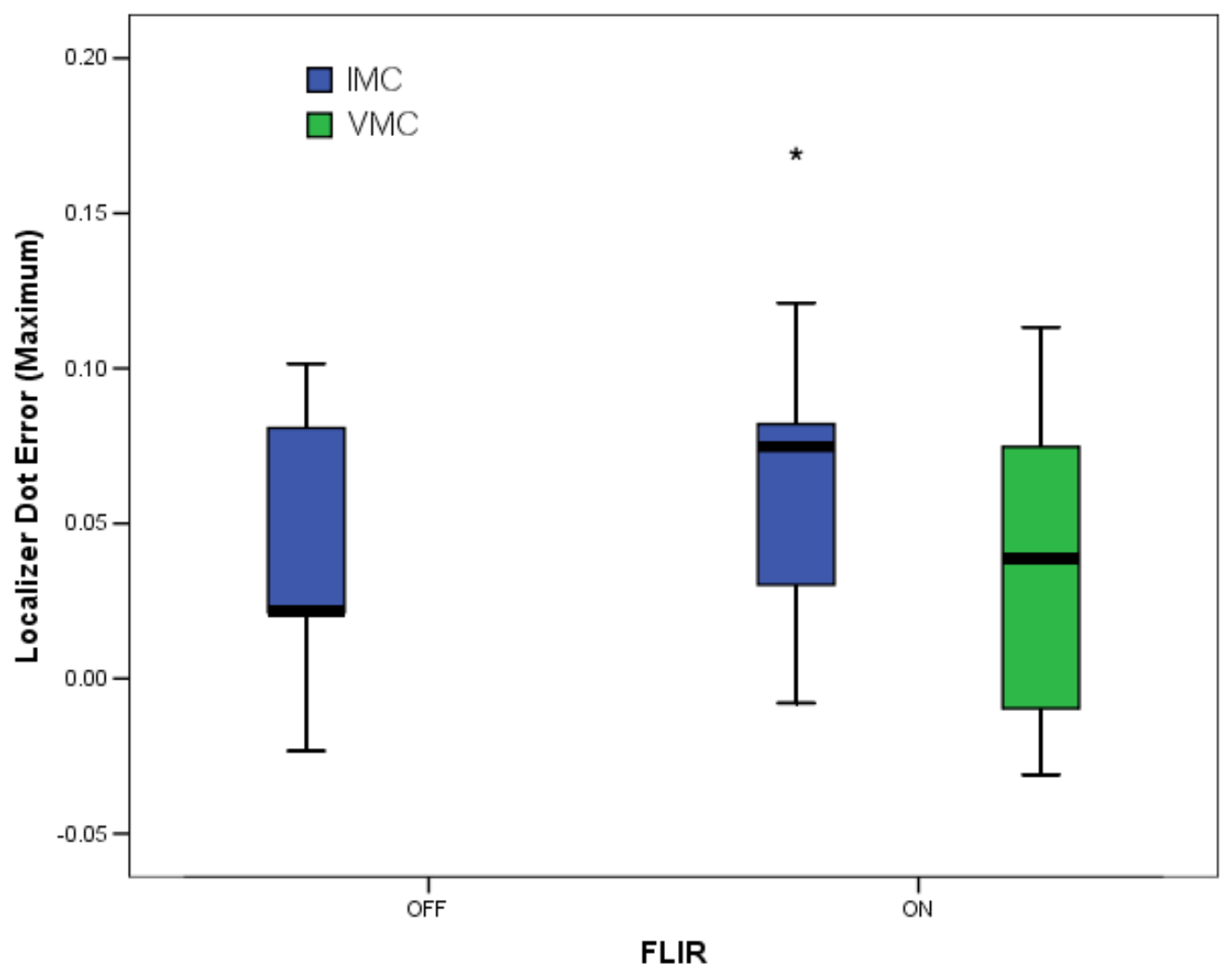

Figure 5: Localizer Dot Error (Maximum Value) During Approach for FLIR (on/off) and during IMC \& VMC conditions

The data in Figure 6 shows that the evaluation pilots were able to track the glideslope within $+/-1$ dot since the maximum excursion was less than $+/-1$ dot on average and the $75^{\text {th }}$ percentile maximum excursion was within this value. One outlier of 2.0 dots occurred in the FLIR On, IMC condition.

A number of testing criteria have been examined for use in evaluation and qualification of FTE. Advisory Circular (AC) 120-29, Appendix 2, Paragraph 6.2.1 provides an excellent candidate for evaluation of approach performance using these displays. The advisory circular defines minimally acceptable performance for approaches from the final approach fix (e.g., $1000 \mathrm{ft}$. HAT) to $200 \mathrm{ft}$. HAT in terms of localizer $(<2 / 3$ dots or $1 / 3$ full scale deflection) and glideslope tracking $\left(<1\right.$ dot or $1 \frac{1}{2}$ full scale deflection). Another useful objective measure of FTE are practical test standards (PTS) which delineate minimally acceptable performance as $<3 / 4$ full scale localizer and glideslope scale deflection between the final approach fix (FAF) and decision height (FAA-S-8081-4D). Both acceptable performance limits are shown in Figure 6 - the PTS (orange, course dash) and the AC 120-29 (red, fine dash) - and illustrate that the maximum glideslope deviation across all runs in both the visual conditions (IMC/VMC) and with both FLIR conditions (on/off) clearly meet the PTS and to the $75^{\text {th }}$ percentile the AC standards. These criteria are not illustrated in Figure 5 (localizer deviation) since both standards are clearly met. 


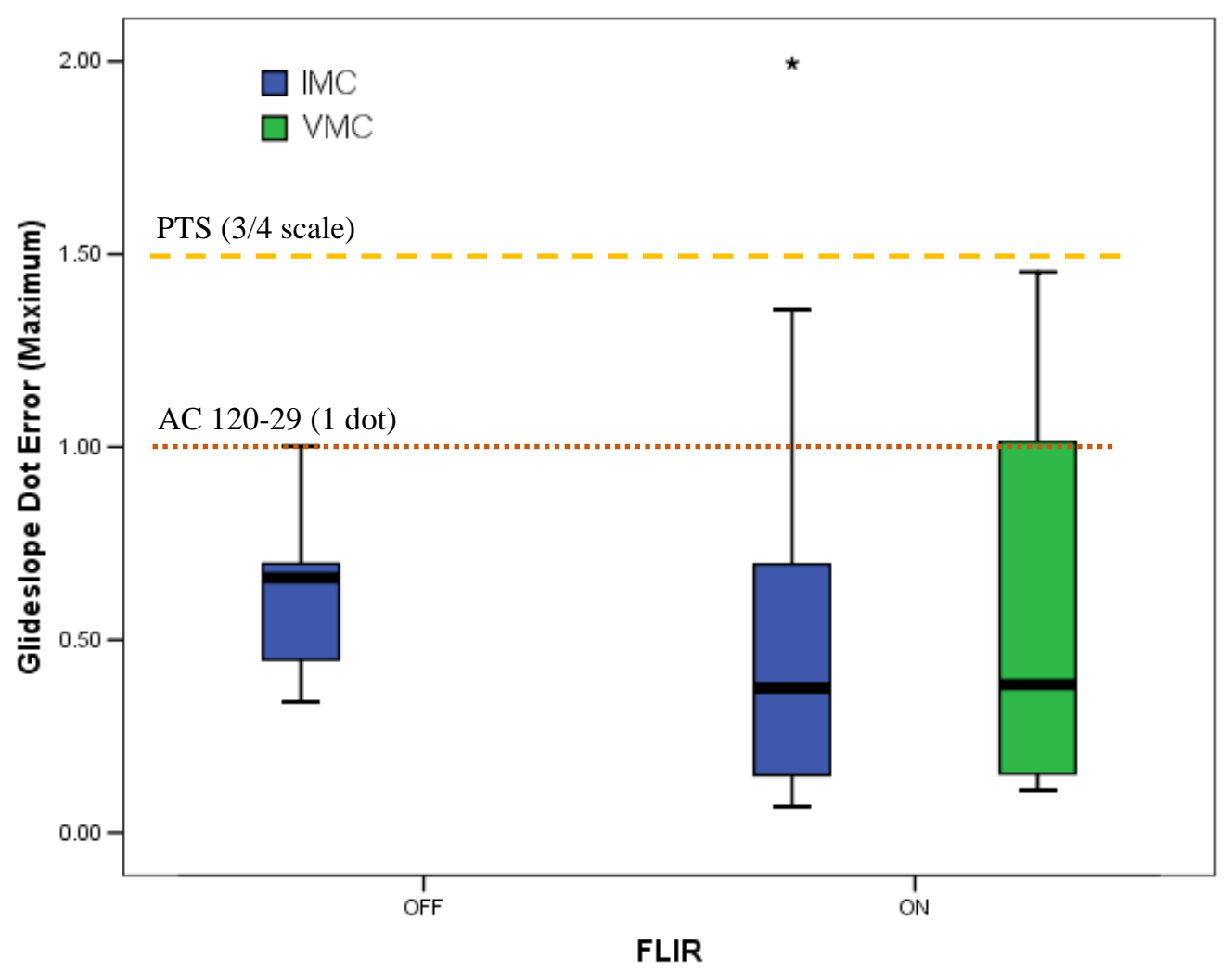

Figure 6: Boxplot Glideslope Dot Error (Maximum Values) During Approach for FLIR (on/off) and during IMC \& VMC conditions

\subsection{Qualitative Analysis}

The data collected also consisted of pilot response data gathered after each trial (post-run) and post-flight. The postrun data included measures of situation awareness, mental workload, and system attributes (comfort, usability, conformality, HUD equivalency, etc.). A 3-point Situation Awareness Rating Technique or SART [10] questionnaire was administered after each run to assess the situational awareness (SA).

An Analysis of Variance (ANOVA) analysis of SART data failed to find a significant effect for FLIR (On, Off), F (1, $18)=0.761, \mathrm{p}=0.394$; or Visibility Condition $(\mathrm{IMC}, \mathrm{VMC}), \mathrm{F}(1,18)=2.424, \mathrm{p}=0.137$. Figure 7 presents the boxplot for SART ratings. The results are directly comparable to a simulation study by Arthur, Prinzel, et al [12]. who reported a range of ' -3 ' (very low SA) to '13' (very high SA) with an average SART rating of '7'; this compares to range of ' 2 ' to ' 13 ' with mean of ' 9 ' for the HWD flight test data reported on here.

Mental workload was assessed using the Air Force Flight Technical Center (AFFTC) Revised Workload Estimation Scale or AFFTC [11]. The 7-point scale that consists of a single number to represent overall workload where ' 1 ' represents "Nothing to do; No system demands" and '7' represents "Overloaded; System unmanageable; Essential tasks undone; Unsafe." The mean workload estimation rating across all visibility and FLIR conditions was a 3 which represents "Moderate Activity; Easily Managed; Considerable Spare Time." Figure 8 presents the boxplot for AFFTC ratings for FLIR and visibility conditions. 


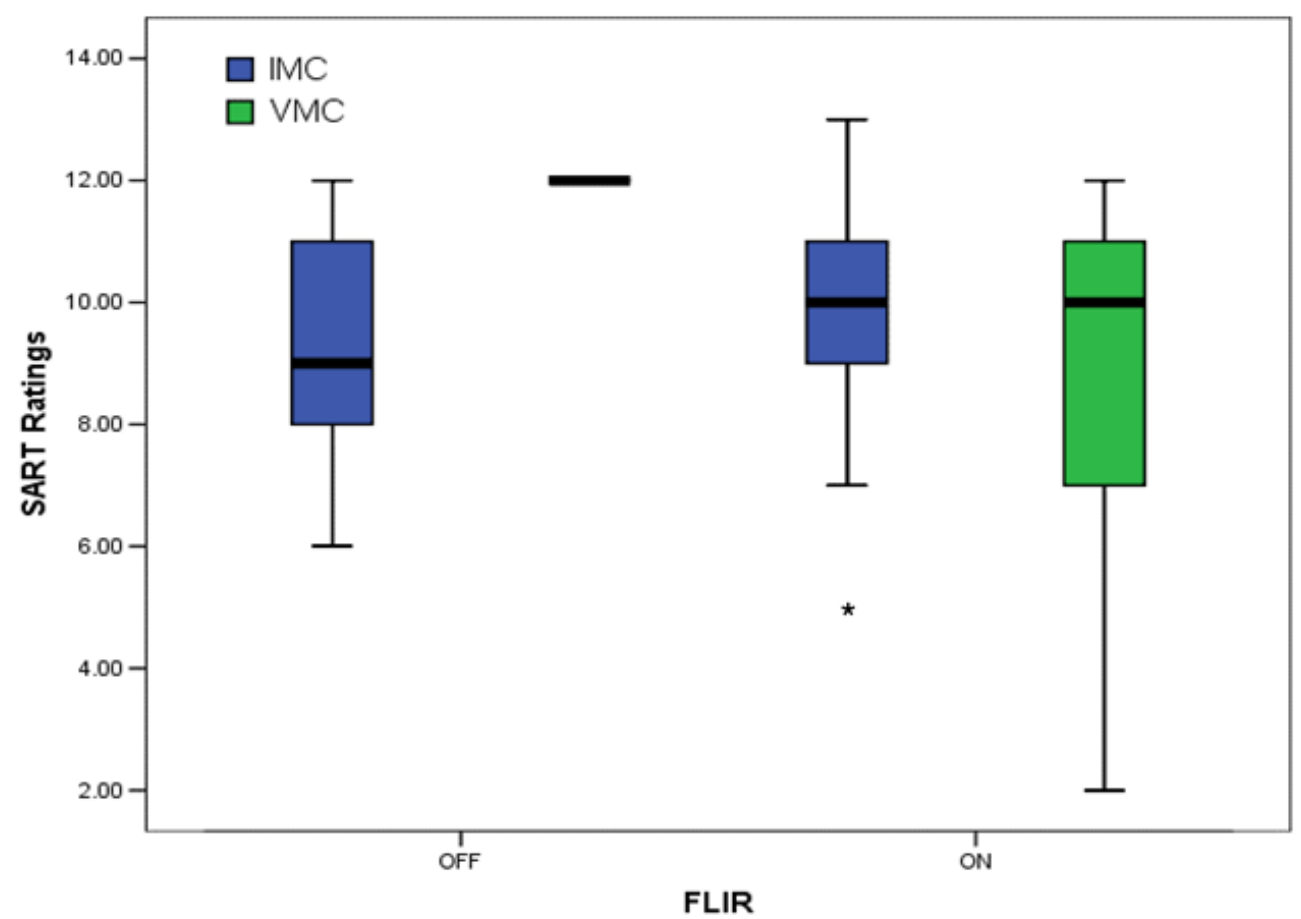

Figure 7: Situation Awareness Rating Technique (SART) Ratings under FLIR and visibility conditions

The statistical results for workload were similar to the findings for SART. No significant effects were found for FLIR, $\mathrm{F}(1,18)=0.795, \mathrm{p}=0.384$; or Visibility Condition, $\mathrm{F}(1,18)=3.231, \mathrm{p}=0.089$. Workload ratings averaged ' 3 ' compared to a 2014 simulation study [12] which Arthur, Prinzel, et al. reported an average AFFTC rating of '3.2' across all HWD approaches that corresponds to "Moderate activity; easily managed; Considerable Spare Time".

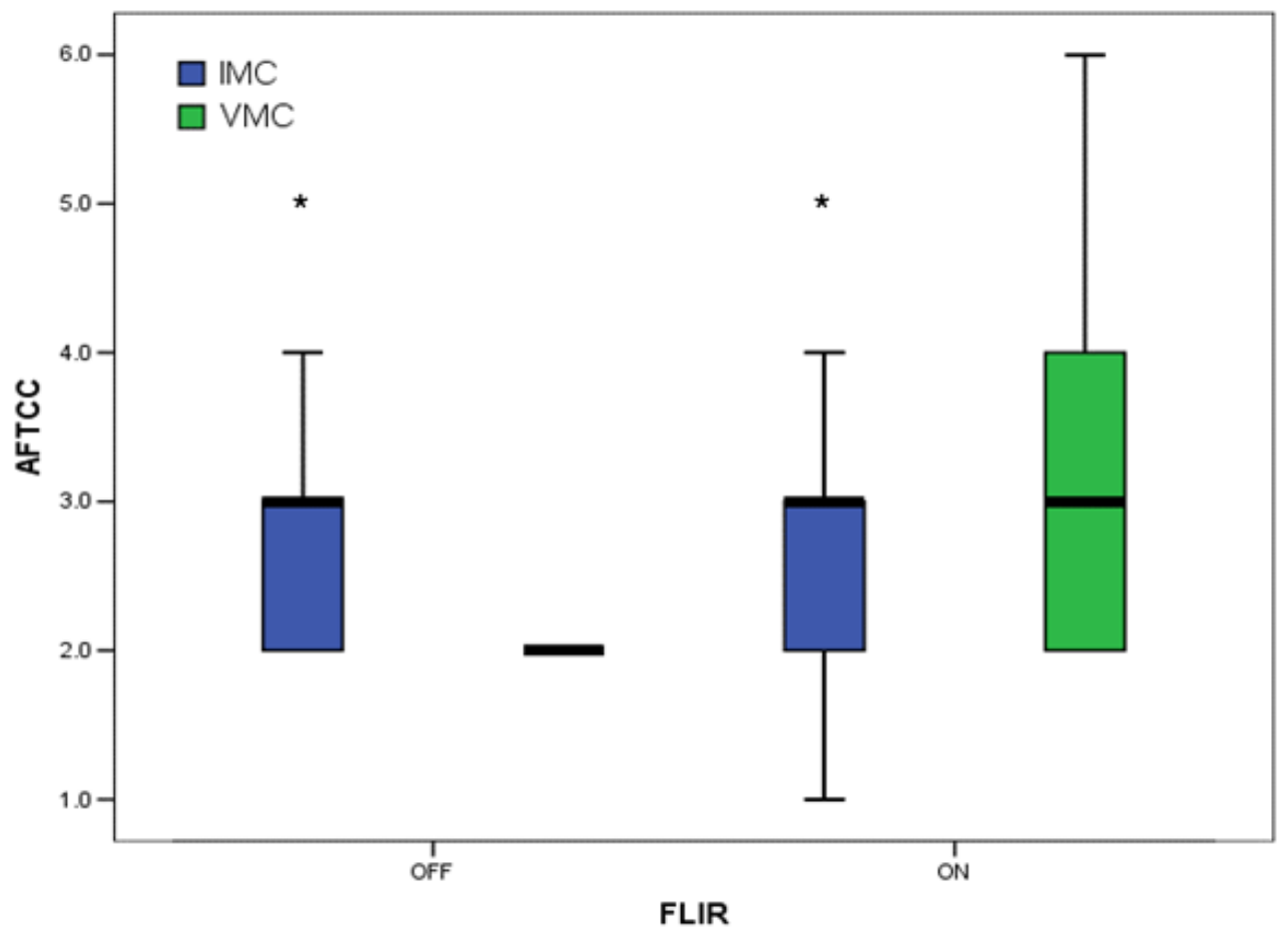

Figure 8: AFFTC Revised Workload Estimation Ratings under FLIR and visibility conditions 


\subsection{Paired Comparisons}

Flight crews were also asked to provide pairwise ratings of situation awareness (SA-SWORD) [13] and "display equivalence" for flight guidance between the HWD and both a HUD (based on past HUD experience since a HUD was not used in this flight test) and head-down (HDD) primary flight display (HDD-PFD). Geo-means were calculated based on the ratings and subsequent parametric statistics [14]. The result for SA-SWORD was a significant main effect, $\mathrm{F}(2,12)=29.377, \mathrm{p}=0.001$. Post-hoc within-subject contrasts revealed that pilots significantly rated the HWD as better for situation awareness (for approach operations) than both the HUD and baseline display standard. (The HUD was also rated significantly better than the head-down display.)

\subsection{Qualitative Display Attribute Ratings}

Results from the post-run and post-flight questionnaires are charted in Figure 9, and Figure 10 using the box plot format. Answers were given for the post-run questions on a 7-point Likert scale and post-experiment 5-point Likert scale. No statistically significant results were found $(\mathrm{p}>0.05)$ for the post-run questionnaire except for ratings of symbology clarity. A rating of ' 1 ' was "strongly disagree", '4' was "neither agree nor disagree", and '7' was "strongly agree."

The post-run questionnaire asked pilots to provide a rating of agreement to several comfort statements:

- The HWD caused me to experience eye strain: Eye strain was not prevalent although the ratings spanned the value of 1 (strongly disagree) to 4 (neutral).

- The HWD caused me to experience headaches: The data indicated that the HWD did not cause any headaches for the EPs (all ratings of 1 and 2, with one outlier being the exception).

- The HWD was comfortable to wear: For the qualitative assessment of comfort, the ratings spanned the entire range of the scale. Although the data tended toward the 'comfortable' side, the median rating was neutral. The HWD was found on occasion to be comfortable and for others, they strongly disagreed and found the HWD uncomfortable.

- The HWD symbols were easy to read (terms of clarity): The qualitative ratings for symbol clarity indicated generally excellent HWD optical performance; with the exception of one outlier, the $25^{\text {th }}$ to $75^{\text {th }}$ percentiles were contained in the 6 and 7 rating range.

- The HWD video/imagery was easy to read (terms of clarity): The qualitative ratings for imagery clarity also indicated generally excellent HWD optical performance; with the exception of one outlier, the $25^{\text {th }}$ to $75^{\text {th }}$ percentiles were contained in the 6 and 7 rating range.

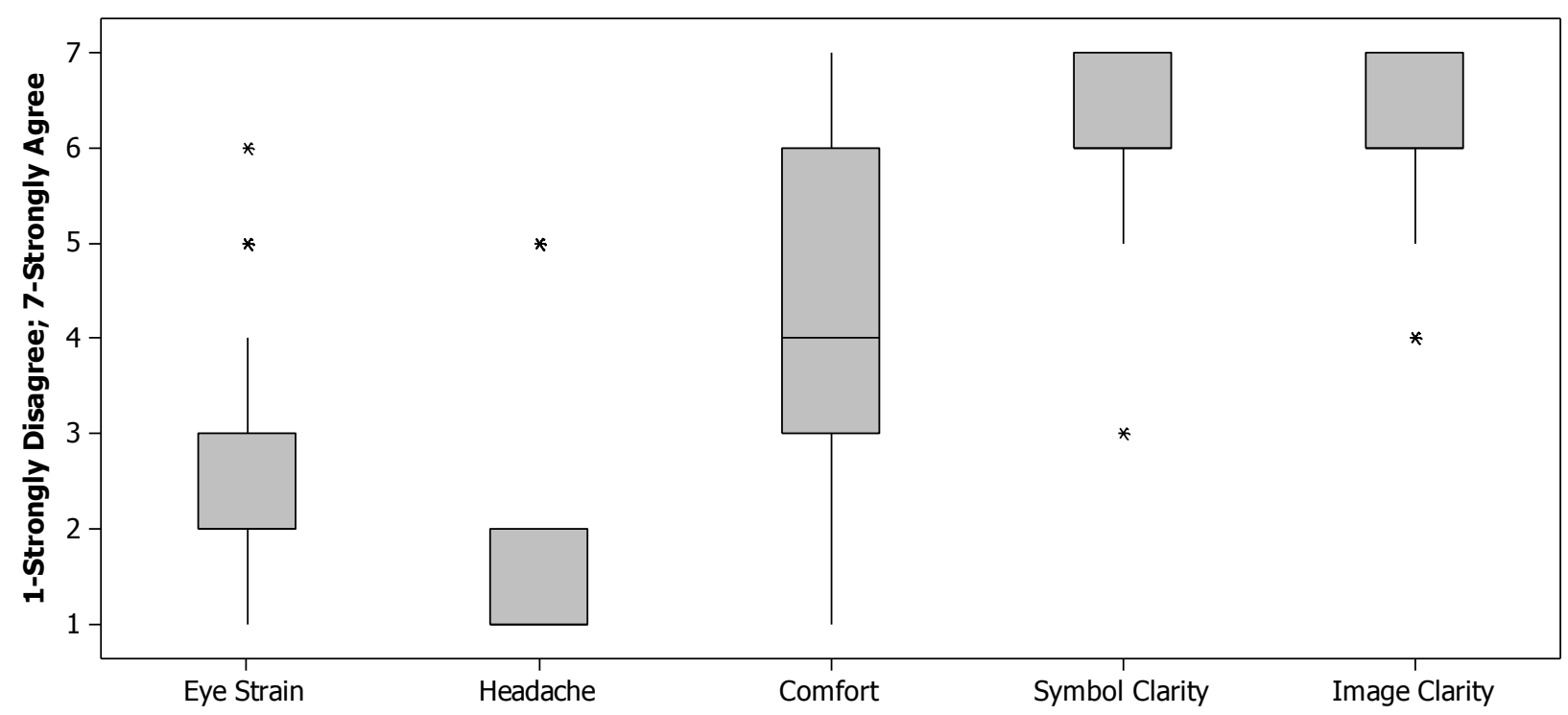

Figure 9: Boxplot of Post-Rung Pilot Response to Comfort Metrics 
Additional post-run questions asked pilots to provide a rating of agreement to usability statements (Figure 10):

- The HWD field-of-view was acceptable to perform task and operation.

- The HWD symbology and imagery was conformal (i.e., aligned and scaled) to the outside world.

- The HWD concept provided usable and sufficient visual cues to safely perform the task/operation.

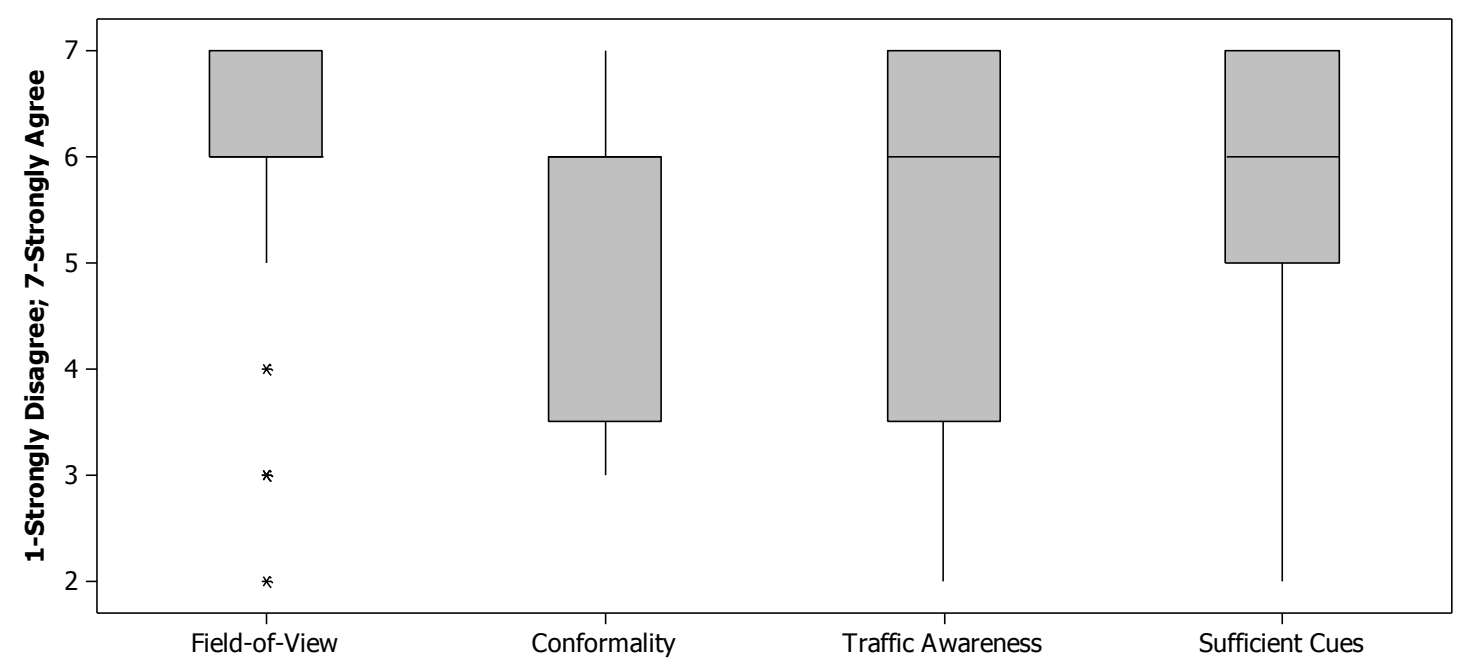

Figure 10: Boxplot of Post-Run Response for Ratings of HWD Usability

These data tended toward the 'good' ratings (i.e., the field-of-view was acceptable) but there was spread in the ratings that warrants further investigation.

HUD Equivalence. Pilots were asked to perform a similar post-experiment paired comparison on the equivalency of the HWD to the HUD based on their experience. Pilots rated the HWD as superior (i.e., equivalent to, if not better than) to the HUD for flight guidance. To supplement the paired comparison, pilots were also asked to provide a rating of agreement to the following statement, "Based on my overall experience with HUDs, I would consider this HWD equivalent to a HUD." The results, using a 1 to 5 Likert scale, were that pilots "agreed" with an average response of ' $3.7^{\prime} /{ }^{\prime} 5.0^{\prime}$ ' (standard deviation 1.15) and a range between 2 and 5 as shown in the boxplot of Figure 11. It is important to note that the flight test did not employ a HUD and, therefore, these ratings are based on the pilots' extensive experience with HUD use. Pilots commented that the HWD, in many ways, had characteristics that provide significant advantages to the HUD.

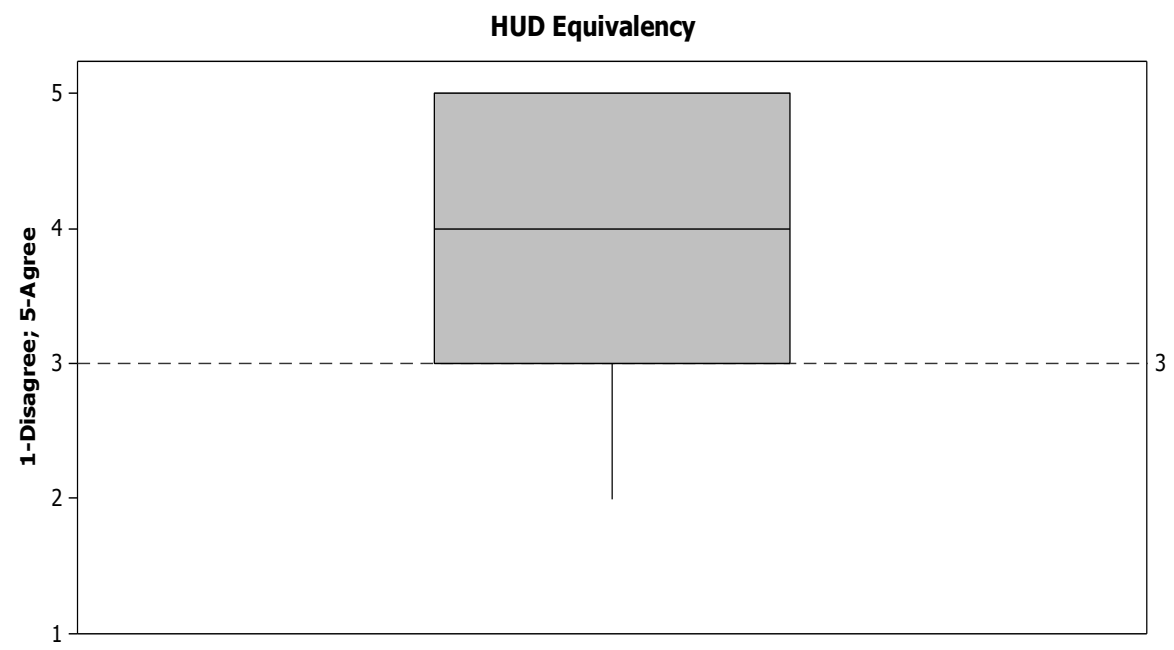

Figure 11: Boxplot of Post-Flight Pilot Response to HUD Equivalency 


\subsection{Discussion}

Quantitative results show that evaluation pilots were able to fly within accepted flight technical error criteria while using the HWD. From the qualitative results and the evaluation pilot comments, important issues came to light. Allowing that test pilots are able to overcome many system limitations and still fly with low flight technical error, additional system improvements are necessary. Several subjects encountered light to moderate turbulence during approaches which combined with system latency and created a "jittery, bouncy" display that was difficult to read and follow. With head-tracked head-up displays, image stabilization is critical for pilot readability and acceptability. Turbulent conditions only exacerbate the image jitter. In the approach run condition of VMC with the FLIR on, latency effects combined with turbulence resulted in increased eye strain, headaches (although minimal), overall discomfort and additional workload. This combination of turbulence and latency is manifested in temporal discrepancies in the conformal image to the actual image. The constant "swimming" of the image in turbulent conditions created an apparent misalignment. For future tests, it would be interesting to compare with an actual HUD in flight under turbulent conditions to tease out head-tracker latency effects. However, when in simulated IMC, the FLIR imagery was not misaligned with the "real world" since it was not visible, and there was little to no reported eye strain even on turbulent approaches. In fact, pilots reported that this configuration reduced workload significantly and subjects reported they were able to focus on pertinent information much easier. Results from the flight test mirror the results in the simulation test [12].

Pilots' lateral performance was well within acceptable standards. The localizer data showed pilots flew within a 0.1 of a dot. Pilot comments said the extended centerline not only helped in lateral line-up on final, but it was a great situational awareness tool on the turn to final. Pilots could track the extended centerline throughout the turn due to the unlimited field-of-regard of the HWD.

Observations and video confirm turbulence was a factor on glideslope performance. EPs were to fly the approach to $200 \mathrm{ft}$. but for a few runs, the safety pilot took over control near $300 \mathrm{ft}$. These close-in maneuvers near the end of the approach resulted in large glideslope deviations (these data have not yet been culled out from the analyses). An additional suspected factor is the single cue pursuit guidance used in the test. (A true flight director was not implemented for the sake of expediency.) The pursuit guidance does not have the same performance as a flight director especially in the final stage of the approach.

The evaluation pilots had many favorable comments with the unlimited-field-of-regard capability of the HWD including that they liked traffic diamonds. They increased situation awareness at minimal expense of lateral field-ofview and increased situation awareness with the extended centerline and the $360^{\circ}$ horizon with heading tick marks. Pilots commented that the traffic diamonds displayed in cyan greatly reduced the clutter of the display. As color is not used on a HUD, on the HWD, it showed value to the pilots as they quickly "disregard" the cyan diamond if it was not currently pertinent to the immediate task. In other words, they could ignore or see the traffic diamond intuitively. In addition, the unlimited field-of-regard allowed pilots to quickly located traffic when identified by ATC. For example, when ATC called traffic at 10 o'clock low, pilots could look to that position and quickly acquire the traffic, something that cannot be done with a fixed HUD.

Several technical challenges were identified with the HWD tested. The absence of peripheral cues in crosswind conditions is a hindrance. This is due to the tested HWD's specific design and should not be a problem with a design tailored to the aviation domain. The system's latency needs to be minimized to reduced imagery jitter. The boresight position needs to be more optimal and better technique is desirable. The traffic diamonds on the HWD demonstrated the de-cluttering effect of the display but further research would be needed to determine if other symbology could also benefit. Another unknown is how colors will appear at different contrast levels when flying in various lighting and weather conditions. (The performance of monochrome green is well known.)

Encumbrance of the HWD is a concern for commercial crews. As the goal is to obtain a sunglasses form factor, this HWD system represented the best analog to that form factor to date. In general, pilots commented that the overall comfort level was acceptable; however, if the test lasted any longer, they felt the HWD would quickly become painful. The HWD system consisted of a strap to keep the HWD stable on the pilots head; however, the tightness of the strap created "hot spots" over time, mainly on the bridge of the nose. Therefore, the HWD system weighing 7 oz. may be too heavy for wearing for more than an hour duration. Reducing the weight of the HWD system continues to be a goal for NASA research. 


\section{SUMMARY}

Though pilots did not fly a HUD in direct comparison of the HWD, pilots felt the HWD was equivalent in terms of flight path performance. The system as tested, within known limitations (brightness, not accommodating glasses, and peripheral view blocked) was able to provide information equivalent to a HUD to allow the subjects to safely taxi and fly ILS approaches in simulated IMC. Quantitative results show performance meets localizer and glideslope evaluation criteria. Qualitative metrics show the evaluation subjects view the prototype HWD as equivalent to a HUD; however, the technology tested had issues that compromise its usability, especially in turbulent conditions. The pilots all liked the HWD's unique capability of unlimited field-of-regard citing: increased situation awareness by use of ADS-B Traffic symbology, the 360 horizon with heading tics, and the extended centerline.

Issues \& Lessons Learned. The nature of POC testing is to discover issues. This initial exploratory flight test proved fruitful as many valuable issues were exposed in flight conditions, especially the effects of wind, turbulence, and various lighting conditions. The performance of the hybrid (optical / inertial) head tracker was generally excellent although data analysis is still on-going to quantify its characteristics. HWD brightness - Not nearly enough, subjects were given clip on glasses to enhance display contrast. The simulated FLIR was useful for testing at minimal expense and installation. The clip-on vision restriction worked well to simulate IMC. Since the NASA test aircraft did not have an operational HUD, future research would benefit from a direct comparison, under identical flight test conditions, of the HUD and HWD.

\section{ACKNOWLEDGEMENTS}

This work was sponsored by NASA's Aviation Safety Program (AvSP), Vehicle Systems Safety Technologies project (Mr. Paul Krasa, Project Manager). Special recognition goes to the project pilots Greg Slover, Rick Yasky, Les Kagey and also electronics support from Charles Howell and the avionics department.

\section{REFERENCES}

[1] Flight Safety Foundation, "Head-Up Guidance System Technology - A Clear path to Increasing Safety," 2009 November.

[2] J. W. Rustenburg, D. O. Tipps and D. Skinn, "A Comparison of landing Parameters from Manual and Automatic landing os Airbus A-320 Aircraft," University of Dayton Research Institute Technical Report UDR-TM-200100003, 2001 November .

[3] R. E. Bailey, L. J. Kramer and S. P. Williams, "Enhance Vision for All-Weather Operations under NextGen," in SPIE 7689, Enhanced ans Synthetic Vision, Orlando, FL, 2010.

[4] L. J. Kramer, R. E. Bailey, K. K. Ellis, R. M. Norman, S. P. Williams, J. J. Arthur III, K. J. Shelton and L. J. Prinzel III, "Enhanced and Synthetic Vision for Terminal Maneuvering Area NextGen Operations," in SPIE Defense, Security, and Sensing, Enhanced and Synthetic Vision Conference, Orlando, 2011.

[5] J. Croft, "Hold Your Head Up," Flight International, pp. 54-56, 27 April 2010.

[6] R. E. Bailey, K. J. Shelton and J. J. Arthur III, "Head-Worn Displays for NextGen," in SPIE Defense, Security, and Sensing, Orlando Fl, 2011 April.

[7] FAA Advisory Circular, "AC 90-106 Enhanced Flight Vision Systems," Federal Aviation Administration, 2010.

[8] Society of Automotive Engineers, "Minimum Performance Standard for Airborne Head Up Display (HUD), SAE-AS-8055," March 1999.

[9] Society of Automotive Engineers, "Transport Category Airplane Head Up Display (HUD) Systems, SAE-ARP5288," May 2001. 
[10] R. M. Taylor, "Situational Awarness Rating Techinique (SART): The development of a tool for aircrew," in Advisory Group for Aerospace Research and Development NATO AGARD-CP-478, Copenhagen, Denmark, 1990.

[11] L. L. Ames and E. J. George, "Air Force Flight Technical Center Revised Workload Estimation Scale," Air Force Flight Technical Center: AFFTC-TIM-93-01, Edwards Air Force Base CA, 1993.

[12] J. J. Arthur III, L. J. Prinzel III, S. P. Williams, K. K. Ellis, D. R. Jones and R. E. Bailey, "Evaluation of a HeadWorn Display System as an Equivalent Head-Up Display for Low Visibility Commercial Operations," NASA Technical Paper, In Press, 2014.

[13] M. A. Vidulich and E. R. Hughes, "Testing a Subjective Metric of Situation Awareness," in Human Factor Society 35th Annual Meeting, Santa Monica, CA, 1991.

[14] T. L. Saaty, The Analytical Hierarchy Process, New York: McGraw-Hill, 1980. 